\title{
Discontinuation of BCG Vaccination Precedes Significant Drop in Type 2 Diabetes in Japanese Children. Role of Inflammation and Cortisol Activity as a Cause of Type 2 Diabetes
}

\author{
John B. Classen*
}

Classen Immunotherapies Inc., 6517 Montrose Avenue, Baltimore, MD 21212, USA

\begin{abstract}
BCG immunization was routinely given to elementary and junior high school students in Japan until it was abruptly discontinued after 2002. Data on the incidence of type 2 diabetes was analyzed to see if there was a change following discontinuation of BCG. The incidence of type 2 diabetes dropped by over $50 \%$ in children from Tokyo following discontinuation of BCG immunization, the relative risk reached 2.78 (95\% confidence interval $1.03-7.480)$. This drop appears to be more significant given the epidemic of type 2 diabetes in children around the world. The increased secretion of cortisol following immunization is thought to result in metabolic syndrome and type 2 diabetes. Japanese children produce larger amounts of cortisol following immunization than White children. This increased production of cortisol may explain why Japanese children have a high risk of type 2 diabetes but low risk of type 1 diabetes compared to White children. The proposed mechanism may explain the epidemic of obesity which starts in children under 6 months of age.
\end{abstract}

Keywords: Type 1 diabetes, type 2 diabetes, BCG, vaccine, cortisol, inflammation.

\section{RESEARCH DESIGN AND METHODS}

BCG vaccine has been linked to an increased risk of type 1 diabetes [1]. BCG immunization was routinely given to Japanese elementary school children age 6 to 7 and junior high school children age 12 to 13 since prior to 1982 . Immunization was discontinued at the end of 2002 [2]. BCG immunization rates were provided by The Research Institute of Tuberculosis, JATA. The incidence of type 2 diabetes in all elementary and all junior high school students in Tokyo Japan has been recorded since 1974 [3-5] and verified data has been published through 2004 [5]. Data on the incidence of type 2 diabetes was analyzed to determine if the incidence declined after discontinuation of BCG vaccine. Only incidence data of type 2 diabetes after 1982 was used because the age of BCG immunization changed in 1982 and this may explain the decreased incidence of diabetes prior to this time. Statistics were performed using Epiinfo 2000, CDC, Atlanta GA and a internet based Poisson calculator. A two stratification analysis was performed with one stratification using data from elementary school children and one stratification using data from junior high school students. Both Poisson and the more conservative Mantel-Haensel Weighted Relative Risk calculation was performed and Greenland/Robins Confidence Limits created. The $\mathrm{P}$ values with both methods were less than 0.05 , with the later being less significant.

\section{RESULTS}

The results (Table 1) show that BCG immunization was associated with an relative risk of $2.78(1.03<\mathrm{RR}<7.48)$.

*Address correspondence to this author at the Classen Immunotherapies Inc., 6517 Montrose Avenue, Baltimore, MD 21212, USA; Tel: 410-3778526; E-mail: Classen@vaccines.net

\section{CONCLUSIONS}

The incidence of type 2 diabetes is increasing throughout the world in children [6] which makes the statistically significant drop in the incidence of type 2 diabetes in Tokyo so remarkable. Several theories have been proposed to explain the epidemic of type 2 diabetes. The obesity theory [7] holds that poor diet and lack of exercise are the cause for type 2 diabetes. A second theory postulates that increased inflammation [8] and the release of cortisol [9] are causing the epidemic of type 2 diabetes. The present data is consistent with inflammation as the cause of the type 2 diabetes epidemic in children.

The theory that poor diet and lack of exercise are causing the epidemic of type 2 diabetes in children has several short comings. The theory does not explain why obesity is increasing drastically in children under 6 month of age [10], who were never very active and don't eat fried foods or other junk food. It also does not explain the simultaneous epidemic of type 1 diabetes [11], which occurs primarily in thin patients. The cortisol theory $[9,12,13]$ was based on the observation that small increases in cortisol activity are associated with metabolic disturbances including increased glucose levels, insulin resistance, increased blood pressure, obesity and hyperlipidemia [14]. Metabolic syndrome and related type 2 diabetes resemble mild Cushingoid Syndrome $[15,16]$. However, the cause for the increased cortisol secretion had not been previously identified even though increased inflammation, which increases cortisol activity, had been identified $[8,9]$.

The decline in the incidence of type 2 diabetes in Japan can be explained by a recently proposed mechanism of action [17]. The proposed mechanism is that type 1 and type 2 diabetes are polar opposite aberrant responses to immunization and other immune stimulants. Immune stimulation with 
Table 1. Rates of Type 2 Diabetes in BCG Vaccinated and Unvaccinated Children

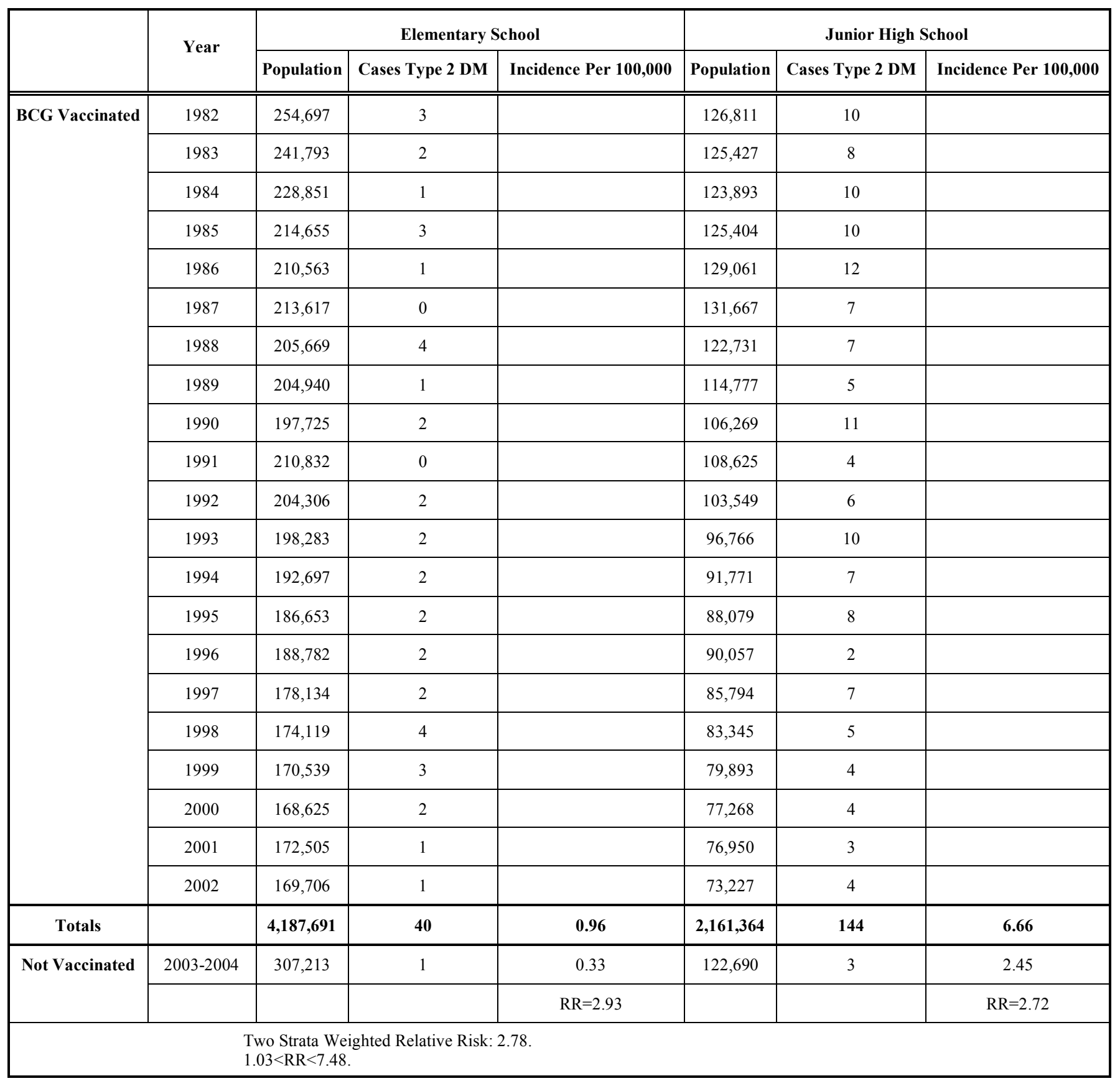

vaccines causes some with low cortisol activity to develop an autoimmune disease including type 1 diabetes $[18,19]$. It is known that decreases in cortisol following adrenalectomy leads to increased rates of type 1 diabetes in mice [20]. In others exposure to immune stimulation activates an neuroendocrine feedback loop to suppress the immune system through increased cortisol activity. Increased cortisol activity leads to type 2 diabetes and metabolic syndrome [9].

Vaccines have been shown to stimulate the immune system and can cause chronic inflammation [21, 22]. Vaccines have been shown to cause cortisol release [23-30]. This can be explained because vaccines cause the release of IL-6 [3133] an cytokine that causes cortisol release [34-36]. Japanese have increased cortisol secretion following immunization compared to Whites [37] and this explains why Japanese have higher rates of type 2 diabetes but lower rates of type 1 diabetes than Whites [4, 11, 38, 39].

This proposed mechanism explains several observations that the obesity theory does not. The vaccine-inflammationcortisol mechanism explains why there is a simultaneous epidemic of type 1 and type 2 diabetes in children. Increased immunization can explain both epidemics. Type 1 diabetes has previously been linked to immunization [18, 19, 40]. BCG vaccine has been previously associated with an increased risk of type 1 diabetes [1] and is now associated with an increased risk of type 2 diabetes. The mechanism explains why the anthrax vaccine is associated with an increased risk of diabetes within 45 days post immunization [41], insulin 
resistance, and greater than 45 days post immunization [41], destruction of islet cells and longer term changes in insulin resistance secondary to cortisol induced obesity. The mechanism explains why those with type 1 diabetes are skinny, lower cortisol activity [20], while type 2 diabetics are obese, higher cortisol activity [9]. It explains why obesity is increasing drastically in children under 6 month of age [10], vaccine induced cortisol activity. It also explains why diet and exercise have not been effective in preventing the epidemic of type 2 diabetes [7].

The observation that discontinuation of BCG immunization was followed by a drop in the incidence of type 2 diabetes needs additional follow up. The incidence of type 2 diabetes in Tokyo varies from year to year. Longer follow up would help better characterize the effect. Studies with other vaccines are also warranted.

\section{ACKNOWLEDGEMENTS}

The author is president and stock holder of Classen Immunotherapies, Inc. which holds patent applications and patents on methods of testing vaccines for causing autoimmunity and diabetes as well as methods of administering vaccines to prevent vaccine induced autoimmunity.

Hitoshi Hoshino, from The Research Institute of Tuberculosis, JATA; 3-1-24 Matsuyama, Kiyose, Tokyo, Japan, provided information on BCG immunization rates of children.

\section{REFERENCES}

[1] Classen DC, Classen JB. The timing of pediatric immunization and the risk of insulin-dependent diabetes mellitus. Infect Dis Clin Pract 1997; 6: 449-54

[2] Hoshino $\mathrm{H}$, Ohmori M, Uchimura $\mathrm{K}$, et al. influences on the adoption of new tuberculosis mass-examination system for school children on tuberculosis of school age. Kekkaku 2005; 80: 475-9.

[3] Urakami T, Kubota S, Nitadori Y, et al. Annual incidence and clinical characteristics of type 2 diabetes in children as detected by urine glucose screening in the Tokyo metropolotan area. Diabetes Care 2005; 28: 1876-81.

[4] Urakami T, Morimoto S, Nitadori Y, et al. Recent change in the annual incidence of childhood type 2 diabetes in the Tokyo metropolitan area. Clin Pediatr Endocrinol 2007; 16: 53-8.

[5] Urakami T, Owada M, Kitagawa T. Recent trend toward the decrease in the incidence of type 2 diabetes in Tokyo. Diabetes Care 2006; 29: 2176-7.

[6] Pinhas-Hamiel O, Zeitler P. The global spread of type 2 diabetes mellitus in children and adolescents. J Pediatr 2005; 146: 693-700.

[7] McAuley KA, Williams SM, Mann JI, et al. Intensive lifestyle changes are necessary to improve insulin sensitivity. Diabetes Care 2002; 25: 445-52.

[8] Pradhan AD, Manson JE, Rifa N, et al. C-reactive protein, interleukin 6 , and risk of developing type 2 diabetes mellitus. JAMA 2001; 286: 327-34.

[9] Bjorntorp P, Holm G, Rosmond R, et al. Hypothalmic arousal, insulin resistance and type 2 diabetes mellitus. Diabet Med 1999; 16:373-83.

[10] Kim J, Petersen KE, Scanlon KS, et al. Trends in overweight from 1980 through 2001 among preschool-aged children enrolled in a health maintenance organization. Obesity $2006 ; 14: 1107-12$

[11] Onkamo P, Vaananen S, Karvonen M, et al. Worldwide increase in the incidence of type 1 diabetes-the analysis of the data on published incidence trends. Diabetologia 1999; 42: 1395-403.
[12] Chiodini I, Torlontano M, Scillitani A, et al. Association of subclinical hypercorticolism with type 2 diabetes mellitus: case control study in hospitalized patients. Eur J Endocrinol 2005; 153: 837-44.

[13] O'Rhally SP. The metabolic syndrome: all in the mind? Diabet Med 1999; 16: 355-7.

[14] Oltmanns KM, Dodt B, Schultes B, et al. Cortisol correlates with metabolic disturbances in a population study of diabetic patients. Eur J Endocrinol 2006; 154: 325-31.

[15] Bjorntorp P, Rosmond R. Hypothalmic origin of the metabolic syndrome X. Ann N Y Acad Sci 1999; 892: 297-307.

[16] Andrew R, Gale CR, Walker BR, et al. Glucocorticoid metabolism and the metabolic syndrome: associations in an elderly population. Exp Clin Endocrinol Diabetes 2002; 110: 284-90.

[17] Classen JB. Type 1 diabetes versus type 2 diabetes/metabolic syndrome. Polar opposites of a spectrum induced by vaccines. Submitted for publication 2007.

[18] Classen JB, Classen DC. Clustering of cases of IDDM occurring 24 years after vaccination is consistent with clustering after infections and progression to IDDM in autoantibody positive individuals. J Pediatr Endocrinol Metab 2003; 16: 495-508.

[19] Classen JB, Classen DC. Clustering of cases of insulin dependent diabetes (IDDM) occurring three years after Hemophilus influenza B (HiB) immunization support causal relationship between immunization and IDDM. Autoimmunity 2002; 35: 247-53.

[20] Saravia-Fernadez F, Durant S, el Hasnaoui A, et al. Environmental and experimental procedures leading to variations in the incidence of diabetes in the nonobese diabetic (NOD) mice. Autoimmunity 1996; 24: 113-21.

[21] Gherardi RK, Coquet M, Cherin P, et al. Macrophage myofasciitis lesions asses long-term persistence of vaccine-derived aluminum hydroxide in muscle. Brain 2001; 124: 1821-31.

[22] Authier FJ, Cherin P, Creange A, et al. Central nervous system disease in patients with macrophage myofasciitis. Brain 2001; 124: 974-83.

[23] Oken E, Kasper DL, Gleason RE, et al. Tetanus toxoid stimulation of the hypothalmic-pituitary-adrenal axis correlates inversely with the increase in tetanus toxoid antibody titers. J Clin Endocrinol Metab 1998; 83: 1691-6.

[24] Catania A, Airaghi L, Manfredi G, et al. Hormonal responses during antigenic challenge in normal subjects. Int J Neurosci 1990; 51: 295-6.

[25] Gunnar MR, Brodersen L, Krueger K, et al. Dampening of adrenocortical responses during infancy: normative changes and individual differences. Child Dev 1996; 67: 877-89.

[26] Lewis M, Ramsay DS. Stress reactivity and self-recognition. Child Dev 1997; 68: 621-9.

[27] Ramsay D, Lewis M. Reactivity and regulation in cortisol and behavioral responses to stress. Child Dev 2003; 74: 456-64.

[28] Lewis M, Thomas D. Cortisol release in infants in response to inoculation. Child Dev 1990; 61: 50-9.

[29] Lewis M, Ramsay DS. Developmental changes in infants' responses to stress. Child Dev 1995; 66: 657-70.

[30] Ramsay DS, Lewis M. Developmental change in infant cortisol and behavioral response to inoculation. Child Dev 1994; 65: 1491-502.

[31] Rowe J, Yerkovich ST, Richmond P, et al. Th-2 local reactions to the acellular diphtheria-tetanus-pertussis vaccine in 4 to 6 year old children. Infect Immun 2005; 73: 8130-5.

[32] Yousfi ME, Mercier S, Breuille D, et al. The inflammatory response to vaccination is altered in the elderly. Mech Ageing Dev 2005; 126: 874-81.

[33] Pourcyrous M, Korones SB, Crouse D, et al. Interleukin-6, Creactive protein, and abnormal cardiorespiratory responses to immunization in premature infants. Pediatrics 1998; 101: e3-8.

[34] Bethin KE, Voght SK, Muglia LJ. Interleukin-6 is an essential, corticotropin-releasing-hormone-independent stimulator of the adrenal axis during immune system activation. Proc Natl Acad Sci USA 2000; 97: 9317-22.

[35] Turnbull AV, Prehar S, Kennedy AR, et al. Interleukin-6 is an afferent signal to the hypothalmo-pituitary-adrenal axis during local inflammation in mice. Endocrinology 2003; 144: 1894-906. 
[36] Vallieres L, Rivest S. Interleukin-6 is a needed proinflammatory cytokine in the prolonged neural activity and transcriptional activation of corticotropin-releasing factor during endotoxemia. Endocrinology 1999; 140: 3890-903.

[37] Lewis M, Ramsay DS, Kawakami K. Differences between Japanese infants and caucasian American infants in behavioral and cortisol response to inoculation. Child Dev 1993; 64: 1722-31.

[38] Ogawa Y, Uchigata Y, Otani T, et al. Proportion of diabetes type in early-onset diabetes in Japan. Diabetes Care 2007; 30: e30.
[39] Dabela D. Incidence of diabetes in youth in the United States. JAMA 2007; 297: 2716-24.

[40] Classen JB, Classen DC. Vaccines and the risk of insulin dependent diabetes (IDDM), potential mechanism of action. Med Hypotheses 2001; 57: 532-38

[41] Joellenbeck LM, Zwanziger LL, Durch IS, et al. The Anthrax Vaccine. Is It Safe? Does It Work? Washington DC: National Academy Press, 2002; 118-179. 